\title{
Breastfeeding up to 12 months of age not associated with increased risk of caries
}

\author{
Abstracted from \\ Tham R, Bowatte G, Dharmage SC, Tan DJ, Lau MX, Dai X, Allen KJ, Lodge CJ. \\ Breastfeeding and the risk of dental caries: a systematic review and meta-analysis. \\ Acta Paediatr 2015; 104: 62-84. \\ Address for correspondence: Caroline Lodge, Allergy and Lung Health Unit (ALHU), \\ Centre for Epidemiology and Biostatistics, School of Population \& Global Health, Faculty of Medicine, \\ Dentistry \& Health Sciences, The University of Melbourne, Level 3, 207 Bouverie Street, \\ Melbourne, Vic. 3010, Australia. E-mail: clodge@unimelb.edu.au
}

\section{Question: Is breastfeeding associated with dental caries?}

Data sources PubMed Central, CINAHL, Embase and reference lists of identified articles.

Study selection Observational and experimental studies published in English where breastfeeding was the exposure and development of caries was the outcome of interest were considered. Two reviewers independently selected studies for inclusion

Data extraction and synthesis Study quality was assessed independently by two researchers using the Newcastle Ottawa Scale (NOS). Key data items, exposure and outcome definitions and effect estimates (odds ratios (OR), relative risks, prevalence ratios) with $95 \%$ Confidence Interval $(95 \% \mathrm{Cl})$ were abstracted where available for inclusion in a meta-analysis. The aim was to assess breastfeeding in two specific time windows; up to 12 months of age and beyond 12 months of age.

Results Sixty-three papers were included. These consisted of 14 cohort studies of which six were nested within RCTs of breastfeeding promotion interventions, three case-control studies and 46 cross-sectional studies. The studies were predominantly conducted in high and middle income countries with only eight studies from low income countries. Forty-six studies were not included in the meta-analysis because of methodological differences in the measures of exposure and outcomes, or reporting of correlational analyses only.

Meta-analysis of one prospective cohort and four cross-sectional studies reported odds ratios for the association between children who were exposed to more versus less breastfeeding up to 12 months $\mathrm{OR}=0.50 ;\left(95 \% \mathrm{Cl} ; 0.25-0.99, \mathrm{I}^{2} 86.8 \%\right)$.

In the two studies which compared ever breastfeeding in the first 12 months with never breastfeeding, both showed a marked protective effect of breastfeeding on dental caries compared with other feeding. Whereas the three studies which compared a longer duration of breastfeeding in the first 12 months to a comparison group which included children who had had some exposure to breastfeeding did not $(34,52,59)$. A meta-analysis of this three study subgroup found $\mathrm{OR}=0.92 ;\left(95 \% \mathrm{Cl} ; 0.69-1.23, \mathrm{I}^{2} 0 \%\right)$

Meta-analysis of two cohort studies, one case-control study and four cross-sectional studies reported odds ratios for the association between more or less breastfeeding after the age of 12 months and dental caries.

Comparison groups for these studies included both those who had never been breastfed and those who had been breastfed for shorter durations. The pooled estimate was OR=1.99; $(95 \% \mathrm{Cl}: 1.35-2.95$, $\left.I^{2} 69.3 \%\right)$.

Meta-analysis of one cohort, one case-control and three crosssectional studies reported odds ratios for the association between more versus less nocturnal breastfeeding and the risk of dental caries amongst the subgroup of children breastfed longer than 12 months. $\mathrm{OR}=7.14 ;\left(95 \% \mathrm{Cl} ; 3.14-16.23, \mathrm{I}^{2} 77.1 \%\right)$.

Conclusions Breastfeeding up to 12 months of age is not associated with an increased risk of dental caries and in fact may offer some protection compared with formula. However, children breastfed beyond 12 months, a time during which all deciduous teeth erupt, had an increased risk of dental caries. This may be due to other factors which are linked with prolonged breastfeeding including nocturnal feeding during sleep, cariogenic foods/drinks in the diet or inadequate oral hygiene practices. Further research with careful control of pertinent confounding factors is needed to elucidate this issue and better inform infant feeding guidelines.

\section{Commentary}

World Health Organization (WHO) guidelines currently recommend exclusive breastfeeding for the first six months with complimentary breastfeeding up to two years of age. The WHO recommendation for exclusive breastfeeding has been in place since 2001. A Cochrane review published in $2012^{1}$ concluded that, 'exclusive breastfeeding for six months (versus three to four months, with continued mixed breastfeeding thereafter) reduces gastrointestinal infection and helps the mother lose weight and prevent pregnancy but has no long-term impact on allergic disease, growth, obesity, cognitive ability or behaviour.' This current review is the most recent of a number of previous reviews that have investigated the relationship between dental caries and breastfeeding. ${ }^{2-6}$

The reviewers have undertaken a broad search in three major databases including a total of 63 studies. The majority of these were cross-sectional studies and the authors provide good detail regarding the quality problems of the included studies. Even in the better quality studies the issue of recall bias was highlighted as a potential problem as most studies gathered data on feeding practices using self-report.

The authors present a narrative synthesis of the bulk of the included studies and also present a number of meta-analyses that include studies of mixed design. These analyses suggest that breastfeeding up to 12 months of age is not associated with an increased risk of dental caries and in may offer some protection 
compared with formula. These findings are similar to those of Avila et al. ${ }^{6}$ who restricted their meta-analysis to the two highest quality cross-sectional studies finding that breast fed children were less affected by dental caries than bottle fed children $(\mathrm{OR}=0.43$, $(95 \% \mathrm{CI}$ : $\left.0.23-.08, \mathrm{I}^{2}: 30.14 \%\right)$.

While the findings of these recent meta-analyses suggest a benefit regarding caries for breastfeeding up to the age of 12 months the quality of the available studies should be taken into consideration. The finding that continuation of breastfeeding beyond 12 months may pose a increased risk of caries is interesting but as the authors point out this may be linked to other associated practices. As noted by the authors in their conclusions, higher quality studies are needed that properly control for confounders to better inform infant feeding practices. Readers might also like to note that a Cochrane review of breastfeeding for oral health is currently ongoing. ${ }^{7}$
Derek Richards

Department of Public Health NHS Forth Valley and Centre for Evidence-based Dentistry, DHSRU, University of Dundee, Scotland

1. Kramer MS, Kakuma R. Optimal duration of exclusive breastfeeding. Cochrane Database Syst Rev. 2012; Issue 8. Art. No. CD003517. DOI: 10.1002/14651858.CD003517.pub2.

2. Valaitis R, Hesch R, Passarellia C, Sheehan D, Sinton J. A systematic review of the relationship between breastfeeding and early childhood caries. Can J Public Health 2000; 91: 411-417.

3. Ribeiro NM, Ribeiro MA. Breastfeeding and early childhood caries: a critical review. | Pediatr (Rio |) 2004; 80: S199-S210.

4. White V. Breastfeeding and the risk of early childhood caries. Evid Based Dent 2008; 9: 86-88,

5. Salone LR, Vann WF Jr, Dee DL. Breastfeeding: an overview of oral and general health benefits. J Am Dent Assoc 2013; 144: 143-151.

6. Avila WM, Pordeus IA, Paiva SM, Martins CC. Breast and Bottle Feeding as Risk Factors for Dental Caries: A Systematic Review and Meta-Analysis. PLoS One 2015; 10 e0142922. doi: 10.1371/journal.pone.0142922. eCollection 2015. PubMed PMID: 26579710; PubMed Central PMCID: PMC4651315.

7. Arora A, Foster JP, Gillies D, Moxey AJ, Moody G, Curtis B. Breastfeeding for oral health in preschool children (Protocol). Cochrane Database Syst Rev 2013; 3: Art. No. CD010416. DOI: 10.1002/14651858.CD010416.

Evidence-Based Dentistry (2016) 17, 75-76. doi:10.1038/sj.ebd.6401183 